\title{
Reynolds number effects on the Reynolds-stress budgets in turbulent channels
}

\author{
Sergio Hoyas ${ }^{a)}$ and Javier Jiménez ${ }^{b)}$ \\ School of Aeronautics, Universidad Politécnica, 28040 Madrid, Spain
}

(Received 28 December 2007; accepted 10 June 2008; published online 31 October 2008)

\begin{abstract}
Budgets for the nonzero components of the Reynolds-stress tensor are presented for numerical channels with Reynolds numbers in the range $\operatorname{Re}_{\tau}=180-2000$. The scaling of the different terms is discussed, both above and within the buffer and viscous layers. Above $x_{2}^{+} \approx 150$, most budget components scale reasonably well with $u_{\tau}^{3} / h$, but the scaling with $u_{\tau}^{4} / \nu$ is generally poor below that level. That is especially true for the dissipations and for the pressure-related terms. The former is traced to the effect of the wall-parallel large-scale motions, and the latter to the scaling of the pressure itself. It is also found that the pressure terms scale better near the wall when they are not separated into their diffusion and deviatoric components, but mostly only because the two terms tend to cancel each other in the viscous sublayer. The budgets, together with their statistical uncertainties, are available electronically from http://torroja.dmt.upm.es/channels.
\end{abstract}

(C) 2008 American Institute of Physics. [DOI: 10.1063/1.3005862]

\section{INTRODUCTION}

The interest of the budget equations for the normal and tangential Reynolds stresses in turbulent shear flows extends from their application in formulating Reynolds-averaged turbulence models to the elucidation of the physics of the cascade. This is especially true in wall-bounded turbulence, where inhomogeneity makes theoretical understanding particularly challenging. Experimental difficulties prevented for a long time the determination of many of the quantities involved, and it was not until the first direct numerical simulation of a turbulent channel ${ }^{1}$ that reliable budgets of the Reynolds-stress tensor could be obtained. ${ }^{2}$ After that pioneering paper, budgets have been published for other numerical flows, ${ }^{3-9}$ but they have usually been limited to relatively low Reynolds numbers by the resolution of the simulations.

The purpose of this paper is to introduce the Reynoldsstress budgets of a recent series of simulations of turbulent channels covering a moderately wide Reynolds number range, ${ }^{10-12}$ and to discuss how they depend on the Reynolds number. It was already noted in Ref. 2 that some dependence was to be expected. It was not clear at the time in which direction, or for which range, although it soon became apparent that the original data at $\operatorname{Re}_{\tau}=180$ were not representative of many of the features of higher Reynolds number flows. ${ }^{9}$ It has been found since then that the classical scaling in wall units of several flow properties near the wall holds only imperfectly, probably up to arbitrarily large Reynolds numbers, ${ }^{13,14}$ and it is interesting to examine how such scaling failures influence the budget equations themselves.

The budget equations are relevant both as diagnostic and as dynamical quantities. As diagnostics, they give information on what has failed when the classical scaling of a Rey-

\footnotetext{
${ }^{a}$ Present address: Univ. Politécnica de Valencia, CMT Motores Térmicos, 46022 Valencia, Spain.

b) Also at Center for Turbulence Research, Stanford University. Electronic mail: jimenez@torroja.dmt.upm.es.
}

nolds stress is found not to hold. As determinants, it is clear that failures in the scaling of the energy equations, or in the budget of the tangential Reynolds stress, bear directly on the dynamics of the flow.

The organization of this paper is as follows. The numerical data sets are introduced in Sec. II, followed by the establishment of the notation for the budget equations. The scaling of the budgets in the outer layers is described in Sec. IV, and the viscous and buffer layers are discussed in Sec. V. A final section concludes. All the budgets discussed in this paper are available in Ref. 15 .

\section{THE NUMERICAL EXPERIMENTS}

Most of the data used in this paper are from the turbulent channel simulations ${ }^{10-12}$ summarized in Table I. We use $x_{j}$ and $u_{j}$, with $j=1 \ldots 3$, for the streamwise, wall-normal, and spanwise coordinates and velocity components, and the kinematic pressure $p$ incorporates the constant fluid density. The density is also left out of the definition of the Reynolds stresses. The channel half width is $h$, and the ${ }^{+}$superindex denotes wall units, defined in terms of the friction velocity $u_{\tau}$ and of the kinematic viscosity $\nu$. Mean quantities are capitalized, and lower-case symbols refer to fluctuations. Primed quantities refer to root-mean-squared (rms) fluctuation intensities. The friction Reynolds number is $\operatorname{Re}_{\tau}=h^{+}$.

The numerical codes ${ }^{1}$ integrate the incompressible Navier-Stokes equations in the form of evolution problems for the wall-normal vorticity $\omega_{2}$ and for the Laplacian of the wall-normal velocity, $\nabla^{2} u_{2}$. The spatial discretization uses Fourier expansions in the wall-parallel planes, dealiased by the two-thirds rule. The three lowest Reynolds numbers use Chebychev polynomials in $x_{2}$, while the highest one uses seven-point compact finite differences. The characteristics of the different simulations with respect to the large scales are documented in the original publications. Their resolution 
TABLE I. Parameters of the simulations. $L_{1}$ and $L_{3}$ are the periodic streamwise and spanwise dimensions of the numerical box, and $h$ is the channel half width. $\Delta_{1}$ and $\Delta_{3}$ are the resolutions in terms of Fourier modes. $N_{2}$ is the number of wall-normal collocation points. $N_{F}$ is the number of fields used for the statistics, and $T$ is their time span.

\begin{tabular}{lccccccrrr}
\hline \hline Case & Line & $\mathrm{Re}_{\tau}$ & $L_{1} / h$ & $L_{3} / h$ & $\Delta_{1}^{+}$ & $\Delta_{3}^{+}$ & $N_{2}$ & $N_{F}$ & $u_{\tau} T / h$ \\
\hline L180 & $\ldots . .$. & 186 & $12 \pi$ & $4 \pi$ & 9 & 6.7 & 97 & 106 & 63 \\
L550 & $-\cdot-$ & 547 & $8 \pi$ & $4 \pi$ & 13 & 6.7 & 257 & 149 & 22 \\
L950 & ---- & 934 & $8 \pi$ & $3 \pi$ & 11 & 5.7 & 385 & 74 & 12 \\
L2000 & - & 2003 & $8 \pi$ & $3 \pi$ & 12 & 6.1 & 633 & 226 & 11 \\
\hline \hline
\end{tabular}

properties regarding the smallest scales are discussed in Ref. 16.

Because of minor bugs discovered in L550 and L950 after the original runs, their statistics have been recomputed by rerunning with a clean code each of the several hundred stored fields, long enough for their statistics near the wall to stabilize. The occasion was used to extend L550, for which relatively few fields had been stored, by several extra eddy turnovers, and to compute the budgets presented here. The difference between the new and the older statistics was found to be negligible except below $x_{2}^{+} \approx 1$, which was the location of the most important bug. The statistics of L180 and L2000 were, as far as we know, bug-free. The behaviors of the turbulent intensities and of the spectra in the buffer layer are discussed in the original publications for the individual simulations, and those in the logarithmic and outer layers are further discussed in Ref. 17.

\section{THE BUDGET EQUATIONS}

Following Ref. 2, the budget equation for the component $\left\langle u_{i} u_{j}\right\rangle$ of the Reynolds-stress tensor is written as

$$
B_{i j} \equiv \mathrm{D}\left\langle u_{i} u_{j}\right\rangle / \mathrm{D} t=P_{i j}+\varepsilon_{i j}+T_{i j}+\Pi_{i j}^{s}+\Pi_{i j}^{d}+V_{i j},
$$

where \langle\rangle stands for averaging over the two homogeneous directions and time. The different terms in the right-hand side of Eq. (1) are referred to as production, dissipation, turbulent diffusion, pressure-strain, pressure diffusion, and viscous diffusion, and are defined as

$$
\begin{aligned}
& P_{i j}=-\left\langle u_{i} u_{k}\right\rangle U_{j, k}-\left\langle u_{j} u_{k}\right\rangle U_{i, k}, \\
& \varepsilon_{i j}=-2 \nu\left\langle u_{i, k} u_{j, k}\right\rangle, \\
& T_{i j}=\Theta_{i j k, k}^{t}=\left\langle u_{i} u_{j} u_{k}\right\rangle_{, k}, \\
& \Pi_{i j}^{s}=\left\langle p\left(u_{i, j}+u_{j, i}\right)\right\rangle, \\
& \Pi_{i j}^{d}=\Theta_{i j k, k}^{p}=-\left[\left\langle p u_{i}\right\rangle \delta_{j k}+\left\langle p u_{j}\right\rangle \delta_{i k}\right]_{, k}, \\
& V_{i j}=\nu\left\langle u_{i} u_{j}\right\rangle_{, k k},
\end{aligned}
$$

where $\delta_{i j}$ is Kronecker's delta, the subscripts ()$_{, j}$ represent derivation with respect to $x_{j}$, and repeated subscripts imply summation over $1, \ldots, 3$. A subscript $K$ refers to the kinetic energy of the fluctuations, $K=\left\langle u_{i} u_{i}\right\rangle / 2$, and $\Theta_{i j k}^{t}$ and $\Theta_{i j k}^{p}$ are diffusion fluxes.
Note that homogeneity along the two wall-parallel directions implies that some terms in Eq. (1) vanish identically for equilibrium channels. Note also that the definitions of $\varepsilon_{K}$ and $V_{K}$ are in part interchangeable, and that the decomposition used above is not the "thermodynamically correct" one. ${ }^{18}$ However, it is known that the difference between the two definitions is negligible everywhere for low Reynolds number channels, ${ }^{19}$ and the same is true for the present ones.

\section{THE OUTER LAYERS}

Away from the buffer layer, the basic scaling of all the terms in Eq. (1) at a fixed $x_{2} / h$ is $u_{\tau}^{3} / h$. In an ideal logarithmic layer, the tangential Reynolds stress would be $u_{\tau}^{2}$, and $U_{1,2}=u_{\tau} / \kappa x_{2}$, where $\kappa$ is the Kármán constant so that the kinetic energy production would be $u_{\tau}^{3} / \kappa x_{2}$. As a consequence, it can be expected that all the terms in Eq. (1) should decrease away from the wall roughly as $x_{2}^{-1}$, and it is convenient to represent them premultiplied by $x_{2}$, as in Fig. 1. With the logarithmic abscissa used in that figure, this has the added advantage of making areas proportional to the integrated energy. Note that the $x_{2}^{-1}$ behavior holds reasonably well for all the budgets in Fig. 1 , from $x_{2}^{+} \approx 50\left(x_{2} / h\right.$ $\approx 0.025)$ to $x_{2} / h \approx 0.4$.

In agreement with previous results, ${ }^{2}$ all the budgets above the buffer layer are dominated by a few terms. Energy enters the flow through the streamwise velocity fluctuations, from where it is extracted by the dissipation and by the pressure-strain term. The pressure redistributes the energy to $u_{2}$ and $u_{3}$, from where it is dissipated. All the other terms are small except above $x_{2} / h \approx 0.4$, where the production decreases because the mean velocity profile flattens, and the dissipation and redistribution terms are compensated by the turbulent and pressure diffusions. The tangential stress, $-\left\langle u_{1} u_{2}\right\rangle$, is generated by the production, and damped by the pressure strain.

In the buffer layer, viscosity becomes important, and the theoretical scale for the terms in Eq. (1) is $u_{\tau}^{4} / \nu$. The limit between the two regions is not sharp, but it can be estimated by testing how the profiles, expressed in outer units, drift as the Reynolds number changes. Those tests cannot be absolute because some change is bound to occur. In an experiment or simulation of only finite accuracy, the only alternative is to test the hypothesis of the drift of a given variable against its estimated numerical and statistical uncertainties. Those can be computed in two different ways. The estimation of the statistical uncertainties of the individual variables 

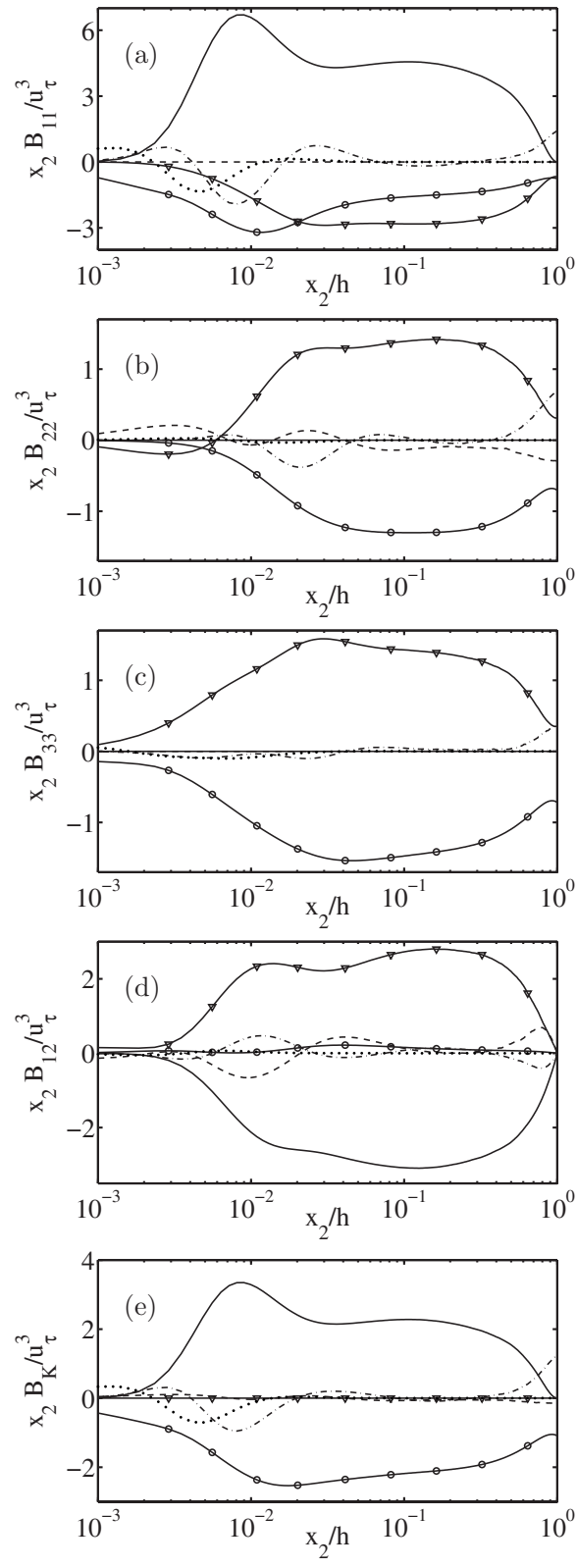

FIG. 1. Premultiplied budgets for the four nonzero Reynolds stresses, and for the total kinetic energy. (-) $P$; (-○一) $\varepsilon ;(-\cdot-) T ;(-\nabla-) \Pi^{s}$; $(---) \Pi^{d} ;(\cdots \cdots \cdots) V$. Case L2000.

is explained in the Appendix. They are due to the limited running time of the simulation, and are of order $10^{-2}$ in the units of Fig. 1. A second error estimate is obtained from the residual balances in Eq. (1), which turns out to be roughly of the same order of magnitude as the statistical uncertainties. Both magnitudes are functions of $x_{2}$ and to test the range of wall distances over which a given scaling can be used, the estimations have to be restricted above some reference length $x_{2}>x_{20}$. When testing for outer scaling, the error typically increases as $x_{20}$ decreases.

What is done in practice is to obtain, for each of the variables within each budget, and for each Reynolds number, a characteristic statistical variance by averaging the square of its statistical uncertainty over the range $x_{2}>x_{20}$. A single number is then obtained for each budget by adding those variances for all the variables in the budget, and for all the
TABLE II. Maximum difference between the premultiplied profiles of the budget terms for the different Reynolds numbers. The last line contains the standard deviation used to normalize each budget, as explained in text. Outer scaling is above $x_{2}^{+}=150$.

\begin{tabular}{lccccc}
\hline \hline & $B_{11}$ & $B_{22}$ & $B_{33}$ & $B_{K}$ & $B_{12}$ \\
\hline$x_{2} P$ & 1.4 & $\ldots$ & $\ldots$ & 1.4 & 4.5 \\
$x_{2} \varepsilon$ & 0.4 & 7.0 & 4.7 & 1.2 & 1.5 \\
$x_{2} T$ & 3.2 & 3.5 & 1.5 & 3.6 & 4.2 \\
$x_{2} \Pi^{s}$ & 1.2 & 8.3 & 4.2 & $\ldots$ & 7.1 \\
$x_{2} \Pi^{d}$ & $\ldots$ & 4.6 & $\ldots$ & 0.5 & 2.8 \\
$x_{2} V$ & 0.1 & 0.2 & 0.4 & 0.2 & 0.01 \\
$\sigma$ & 0.18 & 0.02 & 0.02 & 0.09 & 0.06 \\
\hline \hline
\end{tabular}

Reynolds numbers. A second error estimate is obtained by adding for each budget the squares of its residual balances for the different Reynolds numbers, each of them averaged over the same $x_{2}$ range as above. The final error estimate for each of the five budgets, $\sigma_{i j}^{2}$, is the maximum of those two partial results. The resulting uncertainties are given in the last line of Table II. They are global values for each budget, and should be compared to the characteristic magnitude of each budget as a whole. When the budget magnitude is defined in the same way as for the uncertainties, i.e., as the root mean of the sum of the squares of its constitutive variables, the statistical uncertainty ranges from $4 \%-5 \%$ for $B_{11}$ and $B_{K}$, to $1 \%-2 \%$ for the other three budgets.

The relative drift of a particular variable $Y$ in $B_{i j}$ is defined as the maximum difference between any two profiles at different Reynolds numbers, divided by the relevant standard deviation,

$$
\max _{x_{2}>x_{20}} \frac{\max _{\mathrm{Re}} Y\left(x_{2}, \mathrm{Re}\right)-\min _{\mathrm{Re}} Y\left(x_{2}, \mathrm{Re}\right)}{\sigma_{i j}},
$$

where $\max _{z}$ and $\min _{z}$, are maxima and minima over the set defined by $z$. Those drifts are functions of $x_{20}$, and two examples are plotted in Fig. 2 for each of the three lowest Reynolds numbers in Table I with respect to L2000. The figure shows that the relevant limit is $x_{20}^{+}$, rather than $x_{20} / h$, which agrees with the classical argument that the thickness of the viscous wall region scales in wall units. ${ }^{20}$ The increase in the drift is steepest between $x_{20}^{+}=100$ and $x_{20}^{+}=200$, and we will therefore define the outer region as $x_{2}^{+}>150$, although any comparable limit would do equally well. Note that case

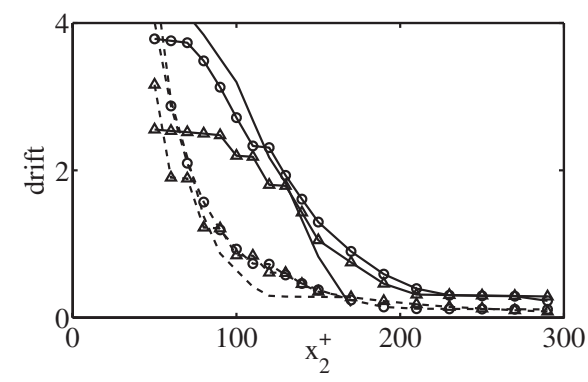

FIG. 2. Drift with respect to L2000 of (-) $x_{2} P_{11}$ and (----) $x_{2} \varepsilon_{11}$, at a fixed $x_{2} / h$, as defined in Eq. (8), as a function of the lower limit $x_{20}^{+}$. Without symbols, L180; $(\bigcirc)$ L550; $(\triangle)$ L950. 
L180 has virtually no outer layer under that definition. All the results below refer only to the drift among the three higher Reynolds numbers in Table I.

The results for all the premultiplied variables in the five budgets are given in Table II. Most of the values in the table are small enough to be consistent with the hypothesis that there is no Reynolds number drift of the components of the outer budgets within the available accuracy. Note however that the table only measures the relevance of the drift of each variable with respect to the particular budget in which it participates. Some of the smaller terms clearly depend on the Reynolds number if they are plotted by themselves and, although their drifts are not strong enough to be significant to the overall result, their scaling failures are physically interesting.

Unfortunately, it is not always possible within the present simulations to decide whether those drifts are low Reynolds number effects, or extend to higher $\operatorname{Re}_{\tau}$. An example is given in Sec. IV A.

\section{A. Turbulent fluxes}

The turbulent- and pressure-diffusion terms are divergences of the vector fluxes $\Theta_{i j k}^{t}$ and $\Theta_{i j k}^{p}$, respectively, whose only nonzero components in a channel are normal to the wall, $k=2$. They are physically interesting because they represent the part of the energy cascade that moves across the inhomogeneous spatial dimension, rather than locally in physical space and across different scales. They may therefore be relevant to how the scales of the turbulent eddies change as they move in $x_{2}$. The fluxes of the kinetic energy $K$ are given in Figs. 3(a) and 3(b). Both are positive above the buffer layer, carrying energy from near the wall to the center of the channel. The pressure fluxes collapse reasonably well in the outer layer, at least for the three highest Reynolds numbers, but the turbulent fluxes do not. Besides a maximum in the buffer region, case L2000 has a second maximum around $x_{2} / h \approx 0.3$, which implies that production slightly exceeds dissipation over the logarithmic layer. A weaker maximum at the same approximate location is present in the highest Reynolds number simulation in Ref. 4, at $\operatorname{Re}_{\tau}=590$, and could perhaps be suggested by the shape of the present case L950.

It is clear that the drift of the $\Theta^{t}$ profiles does not saturate within the range of our simulations, but an outer peak of triple products such as $\Theta_{112}^{t}$ is found in high Reynolds number boundary layers, and it is of some interest to compare the present observations with those experiments.

Figure 3(c) contains $\Theta_{112}^{t}$ above $x_{2}^{+}=150$ from our simulations and from several experimental boundary layers. The experimental data are noisy, especially near the wall. They display the same broad maximum around $x_{2} / h=0.5$ as the simulations, but two of them ${ }^{13,23}$ also contain an intermediate peak, too near the wall to correspond to the outer peak of the simulations, but well outside the buffer layer. For example, the data in Ref. 13, which are especially well resolved near the wall, have a buffer layer peak around $x_{2}^{+}=35$, the intermediate peak visible in Fig. 3(c) at $x_{2} / h \approx 0.1\left(x_{2}^{+} \approx 1000\right)$, and the broad maximum near $x_{2} / h=0.4$. The intermediate
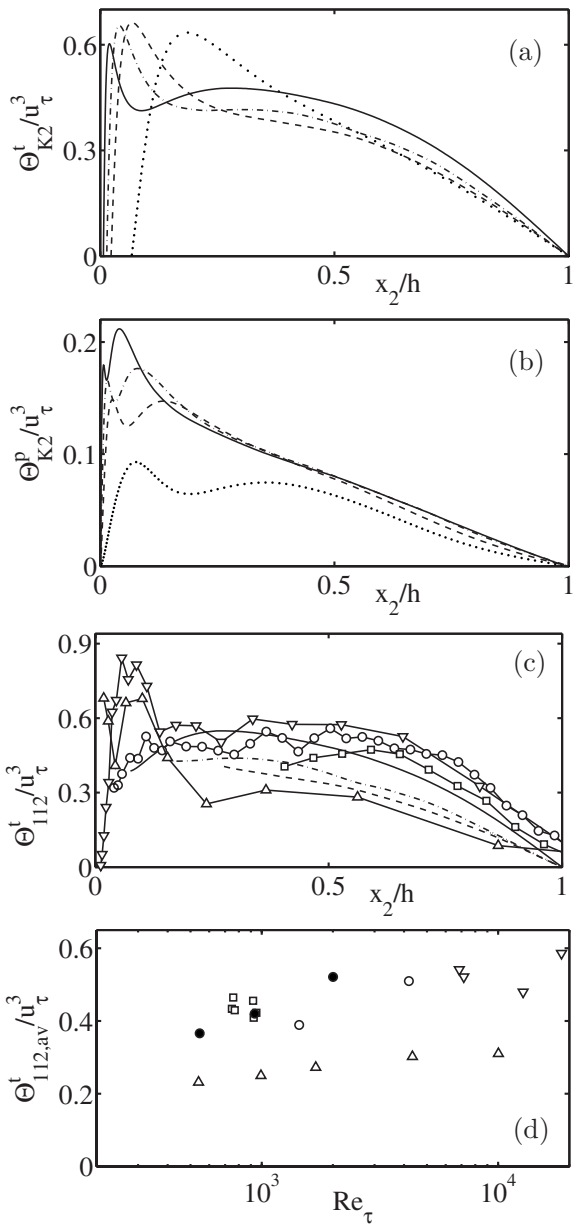

FIG. 3. Kinetic energy fluxes for the numerical channels. Lines are as in Table I. (a) $\Theta_{K^{\circ}}^{t}$ (b) $\Theta_{K^{\prime}}^{p}$ (c) Individual profiles of $\Theta_{112}^{t}$ for several Reynolds numbers, in outer scaling above $x_{2}^{+}=150$. Lines are the numerical channels, while symbols are experimental boundary layers normalized with $h=\delta_{995}$. ( $\square)$ Ref. 21, $\operatorname{Re}_{\tau}=960$; $(\bigcirc)$ Ref. 22, $\operatorname{Re}_{\tau}=4.2 \times 10^{3} ;(\triangle)$ Ref. 13, $\operatorname{Re}_{\tau}$ $=10^{4} ;(\nabla)$ Ref. 23, $\operatorname{Re}_{\tau}=1.8 \times 10^{4}$. (d) Mean value of $\Theta_{112}^{t}$ over $0.3 \leq x_{2} / h$ $\leq 0.5$. Symbols as in (c), but each experiment now includes all the available Reynolds numbers.

peak is not present in the simulations, and it is not explicitly discussed in any of the original experimental publications. Since it is unclear whether it should be considered a high Reynolds number phenomenon, a property of boundary layers not present in channels, or an experimental artifact, we restrict ourselves to the behavior of the broader outer maximum, which is believed to occur at a fixed location in outer units. ${ }^{24}$

Figure 3(d) contains the averaged value of $\Theta_{112}^{t}$ over $0.3 \leq x_{2} / h \leq 0.5$. In the Reynolds number range in which experiments and simulations overlap they agree within experimental noise, and the mean values of the simulations increase with increasing $\operatorname{Re}_{\tau}$. For higher Reynolds numbers, where there are no channel simulations with which to compare, the mean values of the boundary layers are roughly constant, with no obvious Reynolds number trend. Moreover, the data from Ref. 13 are lower than other results at comparable Reynolds numbers, suggesting a lack of universality even among different boundary layers. The reason for this particular discrepancy is unclear. It could perhaps be related 
to the different ways in which the Reynolds number was controlled in that experiment, which was by pressurizing the flow instead of by the more standard methods of varying the measuring station or the tunnel velocity. That amounts to a different tripping mechanism, whose effect on boundary layers is known to be long-lasting. ${ }^{21}$

The end result is that it is impossible to determine either from the simulations or from the experiments whether this particular turbulent flux has a definite high Reynolds number limit. The question is interesting because there is evidence that the fluctuation intensities of the streamwise velocity at fixed $x_{2} / h$ increase in the outer layer with the Reynolds number, ${ }^{12,13}$ but it is unknown whether that trend eventually saturates or continues for arbitrarily high Reynolds numbers. ${ }^{25}$ It is unlikely that a drift of the intensities should not be accompanied by the drift of some of the terms of the corresponding budgets, and a clearer trend of the triple products would have given information on the trend of the intensities.

Other variables suffer from similar ambiguities, which can probably only be resolved by further experiments or simulations. For example, the product $\Theta_{122}^{t}$ is even less universal than $\Theta_{112}^{t}$, and even harder to compare with boundary layers. Not only does it change sign at different locations in the outer layers of the four numerical channels but vanishes at the edge of the boundary layers, and not at the channel center line.

\section{THE BUFFER AND VISCOUS LAYERS}

The wall-scaled budgets within the viscous and buffer layers are given in Fig. 4, normalized in wall units. Contrary to the behavior in the outer layer, most terms are active in this region, ${ }^{2}$ although some of them, such as the viscous diffusion, are only active in the viscous sublayer below $x_{2}^{+}$ $\approx 10$. The normalized drifts for wall-scaled variables below $x_{2}^{+}=100$ are given in Table III, and look different from those in Table II. While the conclusion from that table was that any scaling failure was relatively weak, it is clear from Table III that inner scaling works poorly near the wall, particularly for the dissipation and for the terms connected with the pressure.

Consider first the dissipations shown in Fig. 5(a). The wall-normal component $\varepsilon_{22}$ is negligible near the wall, and only the terms coming from the two wall-parallel velocity components are relevant. Both of them increase with the Reynolds number below $x_{2}^{+} \approx 20$. Figure 5(b) compiles results of the dissipation at the wall from other simulations, and confirms this growth. The behavior at Reynolds numbers not accessible to simulations is less certain. Although there are experimental measurements of the shear-stress fluctuations at the wall, especially of $\varepsilon_{11}$, their scatter is large due to measurement difficulties. ${ }^{28,29}$ The best experimental values from Ref. 29 range from $\varepsilon_{11}^{+} \approx 0.25$ at $\operatorname{Re}_{\tau} \approx 200$ to $\varepsilon_{11}^{+}$ $\approx 0.35$ at $\operatorname{Re}_{\tau} \approx 1000$, and are consistent with Fig. 5(b). It looks from that figure as if there could be an asymptotic leveling of the wall dissipation at higher Reynolds numbers, although that would probably be inconsistent with the known growth with $\mathrm{Re}_{\tau}$ of the near-wall velocity fluctuations. ${ }^{13,30}$ Since $\varepsilon_{11}=-2 \nu\left(\partial_{2} u_{1}^{\prime}\right)^{2}$ at the wall, the drift in the velocity
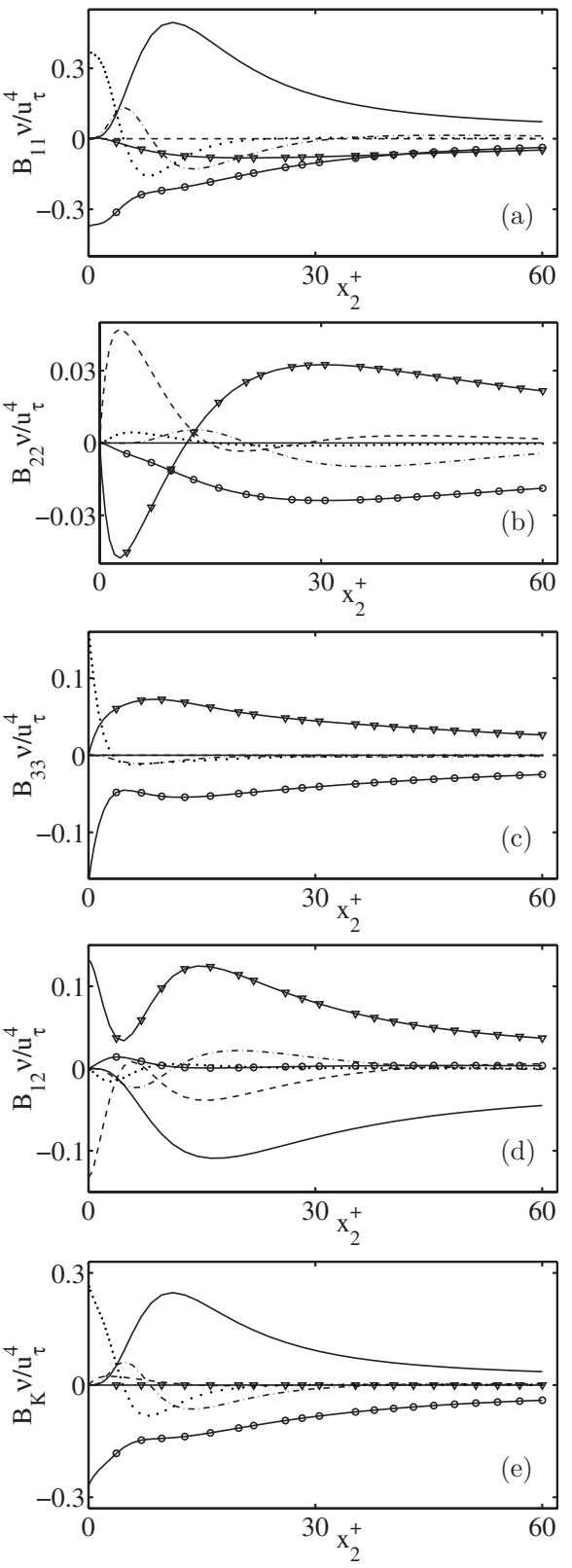

FIG. 4. Budgets for the four nonzero Reynolds stresses, and for the total kinetic energy, in the buffer layer and in wall units. $(-) P$; $(-\bigcirc-) \varepsilon$; $(-\cdot-) T ;(-\nabla-) \Pi^{s} ;(---) \Pi^{d} ;(\cdots \cdots \cdots) V$. Case L2000.

fluctuations probably implies a similar drift in the dissipation.

An interesting data point was obtained for the atmospheric surface layer in Ref. 30. Their measurement of $\partial_{2} u_{1}^{\prime}$ at $x_{2}^{+}=2$ implies $-\varepsilon_{11}^{+} \approx 0.55$ at $\operatorname{Re}_{\tau} \approx 7 \times 10^{5}$, but it drops at the wall to $-\varepsilon_{11}^{+} \approx 0.45$. The extrapolation of the data in Fig. 5(b) would be intermediate between those two values. The lower measurement would imply a leveling of the dissipation at very high $\operatorname{Re}_{\tau}$, while the higher one would imply continued growth. There is no evidence in the $\varepsilon_{11}$ profiles in Fig. 5(b) of any sharp variation of the dissipation between $x_{2}^{+}=2$ and the wall, and the profiles in Ref. 30, although noisy, are consistent with those in Fig. 5(b) up to the last measured point away from the wall. It is unclear from the paper how the wall shear was estimated, but the balance of the evidence 
TABLE III. Maximum difference between the profiles of the budget terms for the different Reynolds numbers. The last line contains the standard deviation used to normalize each budget, as explained in the text. Inner scaling is below $x_{2}^{+}=100$.

\begin{tabular}{lccccc}
\hline \hline & $B_{11}$ & $B_{22}$ & $B_{33}$ & $B_{K}$ & $B_{12}$ \\
\hline$P$ & 17 & $\ldots$ & $\ldots$ & 15 & 7 \\
$\varepsilon$ & 30 & 19 & 41 & 36 & 0.4 \\
$T$ & 20 & 10 & 3 & 18 & 4 \\
$\Pi^{s}$ & 8 & 66 & 45 & $\ldots$ & 12 \\
$\Pi^{d}$ & $\cdots$ & 68 & $\ldots$ & 7 & 5 \\
$V$ & 13 & 4 & 9 & 13 & 0.3 \\
$\Pi^{s}+\Pi^{d}$ & 8 & 29 & 45 & 7 & 10 \\
$\sigma \times 10^{3}$ & 1 & 0.1 & 0.2 & 0.6 & 1.4 \\
\hline \hline
\end{tabular}

suggests that the higher of the two above values is the one to be preferred, implying continued growth.

That the dissipation near the wall should vary with the Reynolds number as a consequence of the large-scale inactive motions was already indicated in Ref. 31. This is supported by Figs. 5(c) and 5(d), which show two-dimensional premultiplied spectra of the total kinetic energy and of the total enstrophy at three heights near the wall. The energy has a "handle" along the upper right-hand end of a line $\lambda_{1} \sim \lambda_{3}$, which is due to large-scale inactive motions coming from the logarithmic layer. ${ }^{10}$ Those modes are irrotational near the wall, ${ }^{32}$ which is why the handle is missing from the two enstrophy spectra farther from the wall. Potential flow, however, cannot satisfy the no-slip boundary condition, and long, wide, and thin viscous layers have to form near the wall to satisfy it. The resulting wall-parallel vorticity is seen in the spectrum closest to the wall in Fig. 5(d), and accounts for the Reynolds number dependence of the dissipation in that region. Note that even if spectra at the wall were not compiled in these simulations, we have already noted that the vorticities at the wall are the wall-normal derivatives of the velocities. The vorticity spectrum at the wall should then be proportional to the energy spectrum, which is seen in Fig. 5(c) to vary little below $x_{2}^{+} \approx 10$ (we are grateful to one of the referees for this observation).

Similar conclusions had been reached by other groups following different lines of reasoning. The simulations in Ref. 26 show the growth of $\varepsilon$ near the wall in the range $\operatorname{Re}_{\tau}=180-640$. Their one-dimensional premultiplied streamwise spectra of the two wall shears collapse well, but the widest wavelengths of the spanwise spectra grow continuously with $\operatorname{Re}_{\tau}$, which the authors attribute to the effect of the large scales. The difference between those two results suggests that the extra energy of their shear fluctuations is contained in modes which are too long, but not too wide, for their computational boxes, which are about $L_{1}^{+}=5000$ and 8000 wall units long for their two highest Reynolds numbers. The shorter of those values is close to the point in Figs. 5(c) and $5(\mathrm{~d})$ where the large-scale handle begins to appear, which is known to scale in wall units. ${ }^{10}$ This suggests that these authors could not observe the streamwise extension of their spectra because of insufficient box length.

The wall-shear spectra from the atmospheric
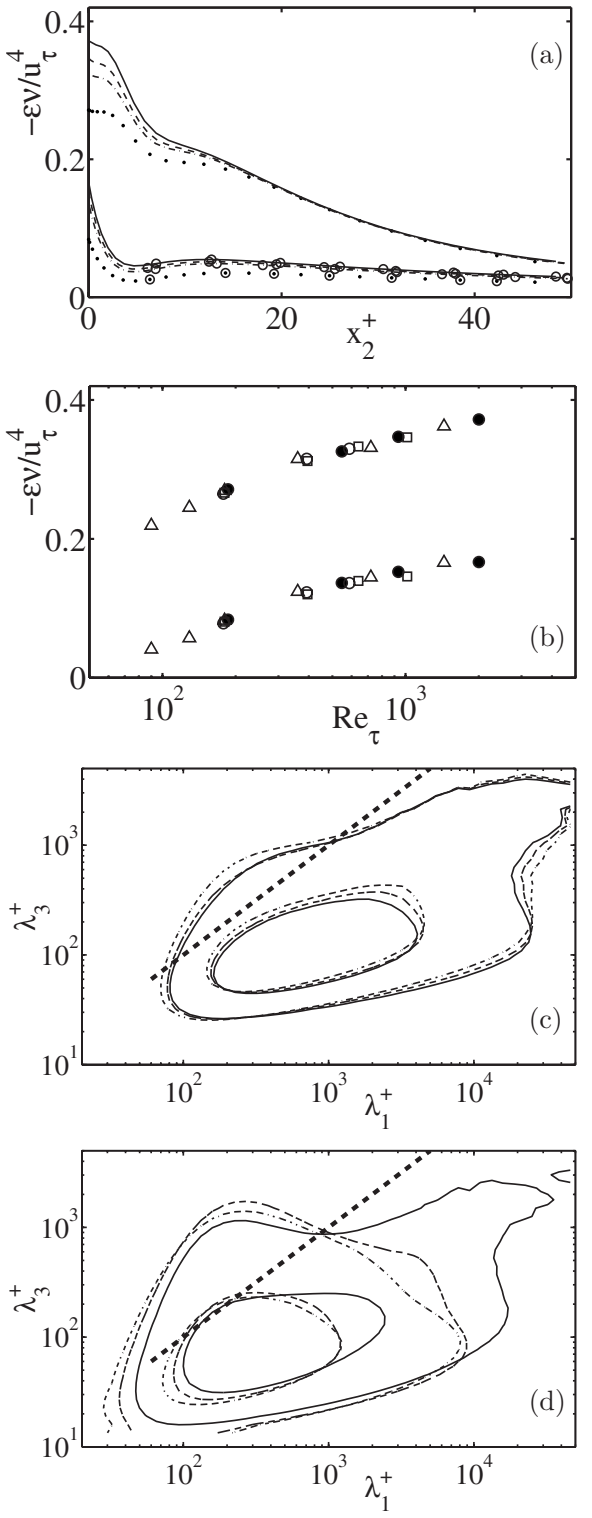

FIG. 5. (a) Reynolds number variability of the dissipation in the buffer layer, Numerical channels. Lines as in Table I. Lines with symbols, $\varepsilon_{33}$; without symbols, $\varepsilon_{11}$. (b) Dissipation at the wall from various simulations. Top data are $\varepsilon_{11}$; bottom ones, $\varepsilon_{33}$. (๑) present channels; $(\bigcirc)$ Ref. 4; ( $\left.\square\right)$ Refs. 26 and 27; $\triangle$ ) Ref. 28. (c) Energy, and (d) enstrophy premultiplied spectra for L2000, at (一) $x_{2}^{+}=6$; (---) $x_{2}^{+}=10 ;(-\cdot-) x_{2}^{+}=14$. The wavelengths are defined in terms of the wavenumbers as $\lambda_{j}=2 \pi / k_{j}$. The two contours for each wall distance contain $50 \%$ and $90 \%$ of the total spectral mass. The dashed diagonal is $\lambda_{1}=\lambda_{3}$.

measurements ${ }^{30}$ grow with Reynolds numbers at all frequencies, but the authors remark that the growth of the spectra of the streamwise shear is strongest for frequencies that, when converted to wavelengths using an advection velocity ${ }^{33} U_{c}^{+}$ $\approx 10$, correspond to $\lambda_{1}^{+} \gtrsim 2000$. This is consistent with Fig. $5(\mathrm{~d})$, and it is interesting that those authors also conclude, on the basis of the absence of "countershear" vorticity near the wall, that the extra dissipation in the viscous sublayer is due to vortex layers, rather than to concentrated vortices.

The scaling failures of the pressure terms are harder to explain because there are no good models for them. ${ }^{2}$ That they scale poorly is clear from Table III and from the two examples in Fig. 6(a), and has been observed in previous 

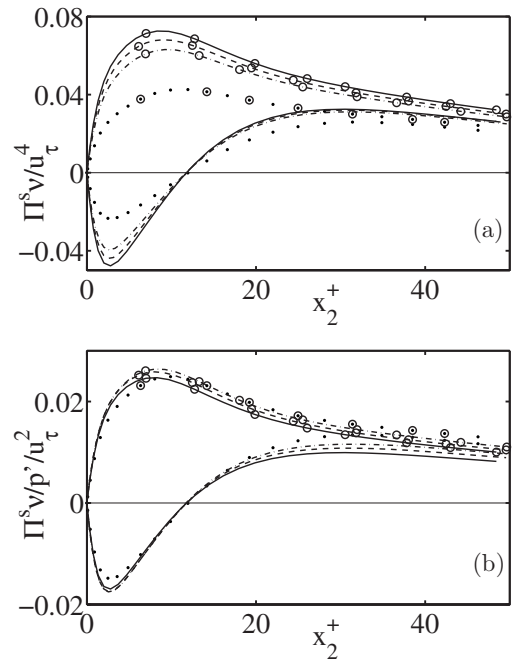

FIG. 6. Reynolds number variability of the pressure-related terms in the buffer layer. Numerical channels. Lines as in Table I. With symbols, $\Pi_{33}^{s}$, and without symbols, $\Pi_{22}^{s}$. (a) Wall scaling. (b) Wall scaling normalized with the rms pressure fluctuation.

simulations at lower Reynolds numbers. ${ }^{7}$ The drift is largest near the wall, and extends farther into the buffer region than in the case of the dissipations. While a good model for these terms is outside the scope of the present paper, the easiest explanation for why the inner scaling does not work is that the pressure itself, which is a global quantity, ${ }^{32,34}$ scales very poorly. On dimensional grounds, the pressure fluctuations should scale everywhere with $u_{\tau}^{2}$, but it is known that the pressure fluctuations at the wall, when scaled in that manner, increase logarithmically with the Reynolds number. ${ }^{28}$ It has been shown more recently that the pressure fluctuation profiles collapse well in outer units far from the wall, ${ }^{35}$ but depend logarithmically on $x_{2}$. When they peak in the buffer layer at a fixed $x_{2}^{+} \approx 30$, the result is that their maximum intensities increase logarithmically. ${ }^{17}$ Independently of the particular model invoked, this strong Reynolds number dependence should be enough to account for any scaling failure of the pressure-related terms in the budgets. In fact, when the pressure-strain terms are normalized in Fig. 6(b) with the local root-mean-squared pressure fluctuation, most of the drift disappears.

It is also interesting that in $B_{22}$ and $B_{12}$, which are the budgets in which the pressure terms can be split into pressure-strain and pressure-diffusion parts, about half of the near-wall drift disappears when the decomposition is not carried out. Table III has an extra line for the sum of the two pressure terms, $\Pi^{s}+\Pi^{d}$, and its drifts are substantially lower than the sum of the two individual contributions. It has been noted before that the choice of how to split the pressure terms is arbitrary ${ }^{36}$ and that the customary separation is not necessarily optimal. That seems to be supported by the present results, but inspection of the profiles in Fig. 4 reveals that the real reason is not that the combined term scales better, but that it is smaller than any of its two components, because the standard decomposition breaks the pressure term into two large contributions of opposite sign that cancel each other almost exactly in the viscous sublayer.

\section{DISCUSSION AND CONCLUSIONS}

We have presented new budgets for the components of the Reynolds-stress tensor in numerical channels, over a fairly wide range of Reynolds numbers. The budgets themselves are available electronically and should be useful both for modeling purposes, and to assist in the understanding of the dynamics of wall turbulence. We have discussed briefly the scaling behaviors of the different terms, both above and within the buffer and viscous layers. We have shown that a reasonable value for the limit between both regions is $x_{2}^{+}$ $\approx 150$. Above that level, most budget components scale well with $u_{\tau}^{3} / h$, although some quantities, such as the triple products, do not scale anywhere within the Reynolds number range of our simulations. Within the buffer layer, on the other hand, the scaling of the budgets with $u_{\tau}^{4} / \nu$ is generally poor. That is especially true of the dissipations and of the pressurerelated terms. We have traced the former to the effect of the wall-parallel large-scale motions, and the latter to the scaling of the pressure itself. We have also noted that a large part of the anomalous scaling of the pressure terms in the buffer and viscous layers disappears when the traditional decomposition into pressure strain and pressure diffusion is not performed, but mostly because the two contributions to the pressure terms tend to cancel each other within the viscous sublayer.

It is reassuring that both within and outside the buffer layer, the budget that collapses best is that of the tangential stress $\left\langle u_{1} u_{2}\right\rangle$. The normal stresses are related to the energy, part of which is due to inactive motions that are not directly involved the momentum transfer. ${ }^{32}$ Neither they nor their budgets are required to scale well in term of $u_{\tau}$. On the other hand, the tangential Reynolds stress is the definition of the friction velocity, and any scaling failure of its budget would have been harder to explain.

\section{ACKNOWLEDGMENTS}

This work was supported in part by the CICYT Contract No. TRA2006-08226, and by the EU FP6 Wallturb Strep under Contract No. AST4-CT-2005-516008. The computations of the budgets were made possible by the generous collaboration of the Port d'Informació Científica (PIC), who lent their mass storage facilities to archive the original raw data, and hosted our postprocessing computers. S.H. was supported in part by the Spanish Ministry of Education and Science, under the Juan de la Cierva program. We are grateful to Messrs. Fernholz and deGraaff for electronic copies of their original data.

\section{APPENDIX: ESTIMATION OF THE VARIANCE}

Consider a data sequence $a(k), k=1, \ldots, N$, where the variable $k$ represents equidistant consecutive instants of time. In our case, the data are usually short-term averages compiled over the intervals $\left(t_{k-1}, t_{k}\right)$. If the different samples were mutually independent, the standard deviation of the overall mean, 


$$
A=N^{-1} \sum_{k=1}^{N} a(k),
$$

could be estimated as

$$
\sigma_{1}=N^{-1 / 2} \sigma_{N}
$$

where

$$
\sigma_{N}^{2}=N^{-1} \sum_{k=1}^{N} a(k)^{2}-A^{2} .
$$

However, since the temporal coherence of the turbulence data is unknown a priori, the samples cannot be assumed to be uncorrelated. We create a sequence of intermediate "coarse-grained" time series

$$
A_{m}(j)=n_{m}^{-1} \sum_{k=j n_{m}+1}^{(j+1) n_{m}} a(k), \quad j=1, \ldots, m,
$$

where $n_{m}=\lfloor N / m\rfloor$, is truncated to the next lower integer. Note that $a \equiv A_{N}$ and $A \equiv A_{1}$, and that disregarding the effect of the truncation of $n_{m}$, the mean value of all the coarse-grained series is $A$. Decreasing the value of $m$ presumably results in more uncorrelated data since their sampling times are more separated. We can then estimate the desired $\sigma_{1}$ as the limit of the coarse-grained approximations

$$
\sigma_{1}=\lim _{m \rightarrow 1} m^{-1 / 2} \sigma_{m}
$$

where $\sigma_{m}$ is the estimated standard deviation of the sequence $A_{m}$. In practice, $\sigma_{m}$ cannot be computed for $m<2$, and Eq. (A5) is evaluated for some decreasing sequence of $m \geq 3$. If all goes well, the expression in Eq. (A5) reaches a plateau well above $m=3$, which is taken as $\sigma_{1}$. Moreover, the absence of a clear limit serves as an indicator that the simulation has not run long enough for a reliable estimation of that variable, and that the standard deviation is underestimated.

${ }^{1}$ J. Kim, P. Moin, and R. D. Moser, "Turbulence statistics in fully developed channel flow at low Reynolds number," J. Fluid Mech. 177, 133 (1987).

${ }^{2}$ N. N. Mansour, J. Kim, and P. Moin, "Reynolds-stress and dissipation-rate budgets in a turbulent channel flow," J. Fluid Mech. 194, 15 (1988).

${ }^{3}$ P. R. Spalart, "Direct simulation of a turbulent boundary layer up to $\operatorname{Re}_{\theta}$ $=1410$,"J. Fluid Mech. 187, 61 (1988).

${ }^{4}$ R. Moser, J. Kim, and N. Mansour, "Direct numerical simulation of a turbulent channel flow up to $\operatorname{Re}_{\tau}=590$," Phys. Fluids 11, 943 (1999).

${ }^{5}$ G. Coleman, J. Kim, and P. Spalart, "Direct numerical simulation of a decelerated wall-bounded turbulent shear flow," J. Fluid Mech. 495, 1 (2003).

${ }^{6} \mathrm{~F}$. Muldoon and S. Acharya, "Analysis of $k-\epsilon$ budgets for film cooling using direct numerical simulation,” AIAA J. 44, 3010 (2006).

${ }^{7}$ H. Abe, H. Kawamura, and Y. Matsuo, "Direct numerical simulation of a fully developed turbulent channel flow with respect to the Reynolds number dependence," ASME J. Fluids Eng. 123, 382 (2001).
${ }^{8}$ J. Komminaho and M. Skote, "Reynolds stress budgets in Couette and boundary layers," Flow, Turbul. Combust. 68, 167 (2002).

${ }^{9}$ R. A. Antonia and J. Kim, "Low-Reynolds-number effects on near-wall turbulence," J. Fluid Mech. 276, 61 (1994).

${ }^{10} \mathrm{~S}$. Hoyas and J. Jiménez, "Scaling of the velocity fluctuations in turbulent channels up to $\operatorname{Re}_{\tau}=2003$," Phys. Fluids 18, 011702 (2006).

${ }^{11}$ J. C. del Álamo and J. Jiménez, "Spectra of very large anisotropic scales in turbulent channels," Phys. Fluids 15, L41 (2003).

${ }^{12}$ J. C. del Álamo, J. Jiménez, P. Zandonade, and R. D. Moser, "Scaling of the energy spectra of turbulent channels," J. Fluid Mech. 500, 135 (2004).

${ }^{13}$ D. B. deGraaff and J. K. Eaton, "Reynolds number scaling of the flat-plate turbulent boundary layer,” J. Fluid Mech. 422, 319 (2000).

${ }^{14}$ M. Metzger, J. Klewicki, K. Bradshaw, and R. Sadr, "Scaling of near-wall axial turbulent stress in the zero pressure gradient boundary layer," Phys. Fluids 13, 1819 (2001).

${ }^{15}$ See: http://torroja.dmt.upm.es/channels

${ }^{16}$ J. C. del Álamo, J. Jiménez, P. Zandonade, and R. D. Moser, "Self-similar vortex clusters in the logarithmic region,” J. Fluid Mech. 561, 329 (2006).

${ }^{17}$ J. Jiménez and S. Hoyas, "Turbulent fluctuations above the buffer layer of wall-bounded flows," J. Fluid Mech. 611, 215 (2008).

${ }^{18} \mathrm{~S}$. Corrsin, "Interpretation of viscous terms in the energy equation," J. Aeronaut. Sci. 20, 853 (1953).

${ }^{19} \mathrm{P}$. Bradshaw and J. Perot, "A note of turbulent energy dissipation in the viscous sublayer," Phys. Fluids A 5, 3305 (1993).

${ }^{20}$ H. Tennekes and J. L. Lumley, A First Course in Turbulence (MIT, Cambridge, MA, 1972).

${ }^{21}$ L. P. Erm and P. N. Joubert, "Low-Reynolds-number turbulent boundary layers," J. Fluid Mech. 230, 1 (1991).

${ }^{22}$ R. W. Smith, "Effect of Reynolds number on the structure of turbulent boundary layers," Ph.D. thesis, Princeton University, 1994.

${ }^{23}$ H. H. Fernholz, E. Krause, M. Nockermann, and M. Schober, "Comparative measurements in the canonical boundary layer at $\operatorname{Re}_{\delta_{2}} \leq 6 \times 10^{4}$ on the wall of the German-Dutch wind tunnel," Phys. Fluids 7, 1275 (1995).

${ }^{24}$ H. H. Fernholz and P. J. Finley, "The incompressible zero-pressuregradient turbulent boundary layer: And assessment of the data," Prog. Aerosp. Sci. 32, 245 (1996).

${ }^{25}$ J. F. Morrison, B. J. McKeon, W. Jiang, and A. J. Smits, "Scaling of the streamwise velocity component in turbulent pipe flow," J. Fluid Mech. 508, 99 (2004).

${ }^{26}$ H. Abe, H. Kawamura, and H. Choi, "Very large-scale structures and their effects on the wall shear-stress fluctuations in a turbulent channel up to $\operatorname{Re}_{\tau}=640$," ASME J. Fluids Eng. 126, 835 (2004).

${ }^{27}$ H. Abe, H. Kawamura, and Y. Matsuo, "Surface heat-flux fluctuations in a turbulent channel flow up to $\operatorname{Re}_{\tau}=1020$ with $\operatorname{Pr}=0.025$ and 0.71 ," Int. J. Heat Fluid Flow 25, 404 (2004).

${ }^{28} \mathrm{Z}$. Hu, C. Morley, and N. Sandham, "Wall pressure and shear stress spectra from direct simulations of channel flow," AIAA J. 44, 1541 (2006).

${ }^{29}$ P. Alfredsson, A. Johansson, J. Haritonides, and H. Eckelmann, "The fluctuating wall-shear stress and the velocity field in the viscous sublayer," Phys. Fluids 31, 1026 (1988).

${ }^{30}$ M. Metzger and J. Klewicki, "A comparative study of near-wall turbulence in high and low Reynolds number boundary layers," Phys. Fluids 13, 692 (2001).

${ }^{31} \mathrm{P}$. Bradshaw, "Inactive motions and pressure fluctuations in turbulent boundary layers," J. Fluid Mech. 30, 241 (1967).

${ }^{32}$ A. Townsend, The Structure of Turbulent Shear Flow, 2nd ed. (Cambridge University Press, Cambridge, 1976).

${ }^{33}$ J. Kim and F. Hussain, "Propagation velocity of perturbations in channel flow,” Phys. Fluids A 5, 695 (1993).

${ }^{34} \mathrm{~J}$. Kim, "On the structure of pressure fluctuations in simulated turbulent channel flow," J. Fluid Mech. 239, 157 (1989).

${ }^{35}$ J. Jiménez and R. D. Moser, "What are we learning from simulating wall turbulence?,” Philos. Trans. R. Soc. London, Ser. A 365, 715 (2007).

${ }^{36}$ J. Lumley, "Pressure-strain correlation," Phys. Fluids 18, 750 (1975). 